\title{
SO HELP ME THE DATABASE
}

\author{
L. Falk and \\ the Editorial Board
}

\section{AN UNCLEAR KRKN ENDGAME POSITION?}

Recently in Sweden, a composition study tournament was held in which the judges were confronted with a particular problem. The study in Diagram 1 (WTM, White to draw) was submitted by A. Gillberg. The judges were faced with the task of evaluating this study.

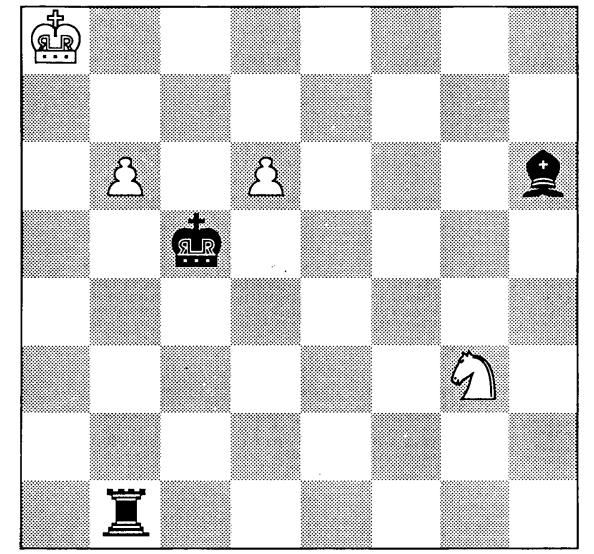

The study's author had suggested the solution as shown; comments by the Editorial Board are within square brackets: 1 . d7! (1. b7? Ra1+ 2. Kb8 Kb6! lost; 1. Ka7? Kc6! lost; 1. Nf5? Kxb6! 2. Nxh6 Rf1! lost) 1. ... Rd1 (1... Bg5? 2. Ne4+ Kxb6 3. Nxg5 Kc7 4. d8=Q+! Kxd8* 5. Ka7 Kc7 6. Ne6+ drawn [an incorrect conclusion, see at ** below]; 1 . ... Kc6!? 2. $\mathrm{d} 8=\mathrm{Q} \mathrm{Ra} 1+3 . \mathrm{Kb} 8 \mathrm{Bf} 4+4 . \mathrm{Qc7}+\mathrm{Bxc7}+5 . \mathrm{bxc7} \mathrm{Rb} 1+6 . \mathrm{Kc} 8$ drawn [still to be checked; so are all KRKN positions to follow]) 2. b7 Bf4 (2.... Ra1+? 3. Kb8 Bf4+ 4. Kc8 Rd1 drawn) 3. Ne4+! (3. b8=Q? Bxb8 4. Kxb8 Rxd7 5. Ne4+ Kc6, with a theoretical win for Black; or 5. Nf5 Rf7 lost; or 5. Kc8 Re7! 6. $\mathrm{Kd8} \operatorname{Re} 5$ !, etc.) 3. ... Kc6! (3. ... Kb6!? 4. b8=Q+! Bxb8 5. Kxb8 Rxd7 6. Kc8 Kc6 7. Nf6 Rf1 8. Ne8 drawn!) 4. Nd6!! (4. b8=Q? Bxb8 5. Kxb8 Rxd7 6. Kc8 Re7 7. Nf6 Re6 [probably] lost), and now: a) 4. ... Ra1+ 5. Kb8 Kxd7 Stalemate! (5. ... Bxd6+? 6. Kc8? [unclear]); b) 4. ... Bxd6 5. b8=Q! (5. d8=Q? Ra1+ [probably] lost) 5. ... Bxb8 6. d8=Q! Rxd8 Stalemate! (6. d8=R!? Bd6 7. Rc8+ Bc7 [probably] lost).

\section{DIAGRAM 1}

** In one of the main variations a $\mathrm{KRKN}$ position arose, marked by *, considered a draw by the composition's author. However, the senior contributor of this note, though impressed with the analysis, had his lingering doubts.

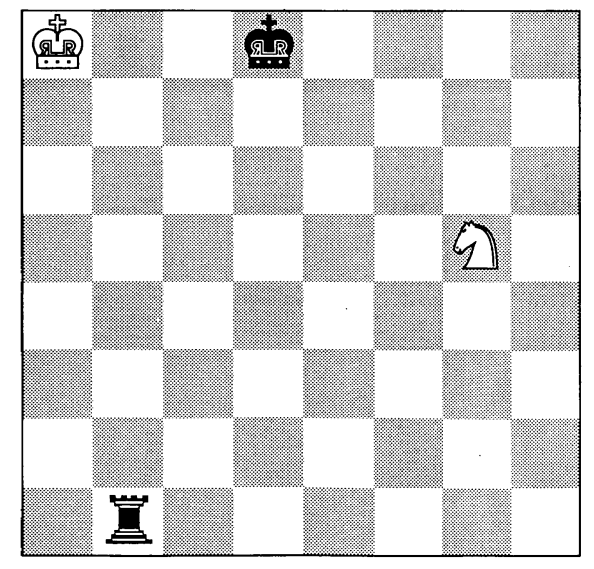

DIAGRAM 2
Although Dr. Falk's impression was that White indeed could draw after $1 . \mathrm{Ka} 7 \mathrm{Kd} 7$ 2. Nf3, no verification was available, not even after invoking the help of Grandmaster Harry Schüssler. Dr. Falk subsequently invoked the help of the databases accessible through the ICCA Journal. 


\section{THE KRKN ENDGAME POSITIONS CLASSIFIED}

Upon this challenge, the Editors activated the generic database system as constructed by Sito Dekker (1988), reachable easily due to the interface constructed by Patrick Schoo. This procedure provided the following results to the senior author.

Contrary to what was suspected by Lars Falk, the position is not a draw but a loss for White. Specifically, it is a mate in 26 moves for Black in the ultimate sense. The ultimate win runs: 1. Ka7 Kd7 2. Nf3 Rd1 3. Kb6 Kd6 4. Nh4 Rb1+ 5. Ka6 Kc6 6. Ka5 Kc5 7. Ka6 Rb6+ 8. Ka7 Rf6 9. Ng2 Rf2 10. Ne1 Kd4 11. Kb6 Ke4 12. Kc6 Re2 13. $\mathrm{Nf3}$ resulting in conversion to a KRK endgame.

The maximum number of moves in the distance-to-conversion metric is 13 . The main variation runs, in agreement with Falk's first three plies, as follows: 1. Ka7 Kd7 2. Nf3 Rd1 3. Kb6 (3. Ne5+ see A below) Kd6 4. Nh4 (4. Ng5 see B below; 4. Kb5 see C below) Rb1+ 5. Ka6 Kc6 6. Ka5 Rf1 7. Ng6 Re1 8. Nh8 Re4 9. Ng6 Kd7 10. Nh8 Re6 11. Ka4 Ke7 12. Ka3 Rh6 etc.

Note the continuations as lettered above, which should be self-explanatory:

A) 1. Ka7 Kd7 2. Nf3 Rd1 3. Ne5+ Kc7 4. Ka6 Rd6+ etc.

B) 1. Ka7 Kd7 2. Nf3 Rd1 3. Kb6 Kd6 4. Ng5 Kd5 5. Kc7 Rg1 6. Nf3 Rg2 7. Ne1 Rg3 etc., or 6. Nh3 Rg3 7. Nf2 Kd4 8. Kd6 Rg2 9. Nh3 etc.

C) 1. Ka7 Kd7 2. Nf3 Rd1 3. Kb6 Kd6 4. Kb5 Kd5 5. Nh4 Rg1 6. Nf3 Rg2 7. Nh4 Rg3 8. Nf5 Rg5 9. Nh6 Ke6 etc.

As a result of this analysis, qualified as "very convincing" by Dr. Falk, the study submitted by Mr. Gillberg was justly disbarred as a composition study for failing its stated purpose viz. White to draw. For the human interest, a few comments by the senior author should be paraphrased below:

"The KRKN endgame seems to be on the borderline between those analyzable as one goes on and those to be entrusted to computers only. It is worth noting that Karpov won this ending from a drawn position in the last round of the Chess Olympiad 1988. He was of course stimulated by his rivalry with Kasparov to achieve a maximum score at whatever cost! Speaking for myself, Gillberg's position would have remained for ever unclear had it not been for the computer's help."

$[\ldots]$

"As I see it, it would be a definite advance in creativity if one were to compose studies using the database and the computer tapping it."

$[\ldots]$

"Thus, the best of computer and human chess could be optimally combined. It also would provide an ideal proving ground for computers in an artistic domain. This is true because no human being would deny study composition to be an artistic exercise, whereas it is sufficiently definable to make it a well-described test field for programming per se."

\section{REFERENCE}

Dekker, S.T. (1988). The program RETRO. A generic database system for endgame positions. Personal Communication. To be published in outline in this Journal. Preliminary versions of the report are available from the author. 